\title{
Alterations in left ventricular volumes induced by Valsalva manoeuvre ${ }^{\mathrm{r}, 2}$
}

\author{
Jeff Z. Brooker, ${ }^{3}$ Edwin L. Alderman, and Donald C. Harrison \\ From the Cardiology Division, Stanford University School of Medicine, Stanford, California 94305, \\ U.S.A.
}

Five patients were studied with left ventriculography during different phases of the Valsalva manoeuvre. Small doses of contrast medium allowed adequate repetitive visualization of the left ventricle for volume calculation. During strain phase, the volume of the left ventricle decreased by nearly 50 per cent in each case, and stroke volume and cardiac output also dropped strikingly. Release of straining was attended by a sharp rebound of left ventricular volume to control levels, with a transient surge of increased cardiac output 42 per cent above that of the resting state.

The Valsalva manoeuvre, when forcefully performed, produces transient but dramatic alterations in venous return, stroke volume, and arterial pressure (Stone, Lyon, and Teirstein, 1965; Goldblatt et al., 1963). The various phases of the Valsalva manoeuvre as defined by Hamilton are associated with well-delineated haemodynamic alterations (Stone et al., 1965; Goldblatt et al., 1963; Hamilton, Woodbury, and Harper, 1936). Phase I, corresponding to the initiation of strain, results in a transient rise in arterial pressure and a rapid decline in venous return. Phase 2, representing the entire duration of the strain phase, has been correlated with falling arterial pressure and increasing heart rate. During the latter portion of this phase, a gradual increase in blood pressure occurs. Phase 3, the abrupt release of the Valsalva manoeuvre, produces a sudden fall of arterial blood pressure and rapid rise of venous return, at a time when the heart rate remains raised. Phase 4 corresponds to the rising blood pressure and pulse pressure seen as the cardiac output increases due to the surge of venous return. In normal subjects, arterial blood pressure and pulse pressure exceed control values, and a reflex bradycardia ensues (Stone et al., 1965; Hamilton et al., 1936).

\section{Received Is January 1974}

1 Presented in part at the Annual Meeting of the American College of Chest Physicians, Denver, Colorado, September 1972.

2 This work was supported in part by an NIH grant and a grant from the National Aeronautics and Space Administration.

3 Supported in part by a Research Fellowship Grant from the Bay Area Heart Research Committee.
Although these classic phases for the circulatory adaptations to the Valsalva manoeuvre have been defined precisely in animals, several measurements have not been feasible in man. For example, previous studies have not reported direct measurement of the alteration of left ventricular volumes during the Valsalva manoeuvre. Techniques for measurement of stroke output and volume measurements during non-steady-state conditions have not generally been available for the study of transient cardiovascular alterations. Approximations have been reported, using the pressure gradient technique (Greenfield et al., 1967), or by evaluating changes in overall cardiac size using metallic epicardial markers (Goldblatt et al., 1963). At Stanford University Medical Center, the development of an angiographic technique for repeatedly measuring left ventricular volume during the course of an intervention in man permitted study of the Valsalva manoeuvre in detail. Thus, the present study was undertaken using this direct angiographic method to measure transient changes in left ventricular chamber size and stroke volume during phases of the Valsalva manoeuvre.

\section{Patients and methods}

Five patients with angina pectoris, including one (Case I) with left ventricular dyskinesis and mild compensated congestive heart failure, and one (Case 2) with subsequent normal coronary arteriograms, were studied during diagnostic cardiac catheterization (Table $\mathrm{I}$ ). The one patient with congestive heart failure was receiving digoxin at the time of the study, and the remaining four 
TABLE I Clinical profile of 5 patients with resting LVEDP before Valsalva manoeuvre

\begin{tabular}{lcclc}
\hline $\begin{array}{l}\text { Case } \\
\text { No. }\end{array}$ & Age & Sex & Clinical diagnosis & $\begin{array}{c}\text { Control } \\
\text { LVEDP* } \\
(m m H g)\end{array}$ \\
\hline I & 50 & M & $\begin{array}{l}\text { Coronary artery } \\
\text { disease, angina, mild } \\
\text { congestive heart failure }\end{array}$ & 27 \\
2 & 59 & F & $\begin{array}{c}\text { Coronary artery } \\
\text { disease, angina }\end{array}$ & 10 \\
3 & 68 & M & $\begin{array}{c}\text { Coronary artery } \\
\text { disease, angina }\end{array}$ & 12 \\
4 & 39 & M & $\begin{array}{c}\text { Coronary artery } \\
\text { disease, angina } \\
\text { Coronary artery } \\
\text { disease, angina }\end{array}$ & 8 \\
\hline
\end{tabular}

LVEDP = left ventricular end-diastolic pressure.

* Resting LVEDP before any Valsalva manoeuvre.

were receiving no medication. Informed consent was obtained from each patient. All patients were fasting and were premedicated with quinalbarbitone, $100 \mathrm{mg}$ intramuscularly. A $125 \mathrm{~cm}$ No. 7 Cournand or Goodale-Lubin catheter was positioned in the pulmonary artery. The end-hole of a $90 \mathrm{~cm}$ Cook polyethylene $5.2 \mathrm{~J}$ catheter was positioned in the descending aorta. Pressures were recorded using a Statham Model P23Db transducer and a photographic multichannel recorder. A Iro $\mathrm{cm}$ Cook polyethylene 6.7 angio-catheter was positioned near the apex of the left ventricle. Serial injections of 76 per cent meglumine and sodium diatrizoate were made during successive phases of the Valsalva manoeuvre (Fig. I). A model IU4A Ensco programmable injector introduced contrast under a pressure of $630 \mathrm{lb} /$ in $^{2}$. Injections were generally initiated $0.2 \mathrm{sec}$ after the patient's QRS and were terminated $0.45 \mathrm{sec}$ later. Using this pressure and this catheter, approximately 9 to $\mathrm{I} 2 \mathrm{ml}$ contrast were injected into the left ventricle during a single mechanical diastole. The time and duration of the injection were indicated on the strip-chart record by means of a syringe position marker.

Patients were studied in the supine position in the posteroanterior projection. End-diastolic and endsystolic volumes were calculated by either manual planimetry of tracings drawn from $35 \mathrm{~mm}$ cine film or by a Hewlett-Packard Model 21 I5A computer, using online data derived from a Tektronics Model 455I light-pen tracing of the video image of the opacified left ventricular chamber. The angiogram was initially recorded on a video-disc recorder at 15 frames per second, and redisplayed on a video monitor for light-pen analysis (Alderman et al., 1973). End-diastolic frames were identified by noting the position of a QRS-triggered mechanical event marker superimposed on the angiographic field. Volumes were not calculated for any beats showing prematurity or aberrancy. Independent cardiac output measurements were obtained during the basal condition, using either standard Fick or indicatordilution methods.

Patients performed the Valsalva manoeuvre by exhaling orally from a full inspiration against a resistance device which included a manometer for monitoring intraoral pressure. Nose clips blocked nasal leakage of air. A $40 \mathrm{mmHg}$ pressure was achieved almost instantly, maintained for 12 to 15 seconds, and then abruptly released.

There were no untoward complications from these angiographic procedures. Though multiple angiograms were obtained during one or two Valsalva manoeuvres, the necessity for high quality studies free of premature beats limits the data to those visualizations obtained during the mid-strain and release phases. These data were obtained in three patients during a single Valsalva manoeuvre, and in two patients from two separate, but
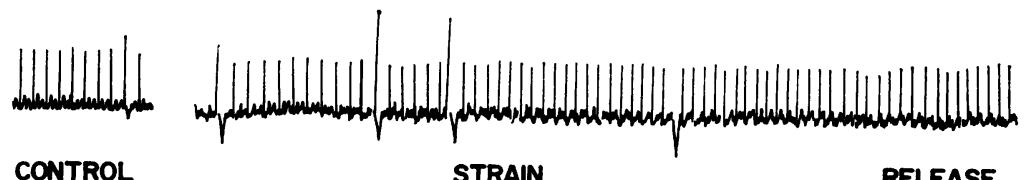

CONTROL

STRAIN

RELEASE

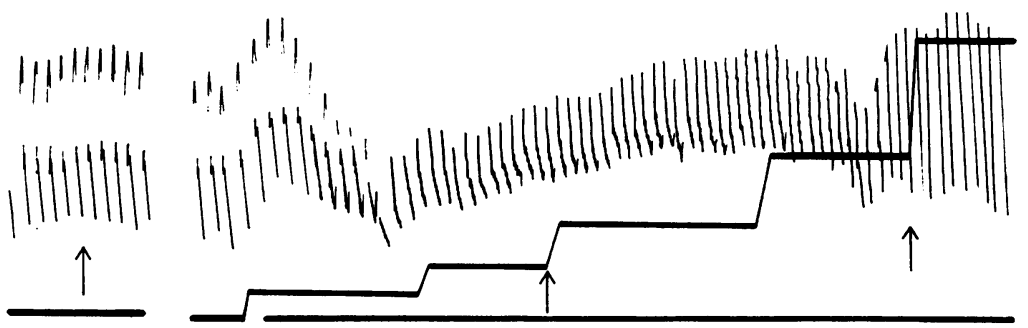

FIG. I Shown from above down are the electrocardiogram, aortic pressure trace, syringe injection marker, and zero pressure baseline during a typical Valsalva manoeuvre. This record was made during the study of Case 2. The upward steps of the syringe position indicator mark the time of separate left ventricular angiograms. Vertical arrows indicate the angiograms used for volume calculation. 
identically performed, manoeuvres. All statistical tests were performed using the paired $t$ test, with significance defined at a $\mathbf{P}$ level of 0.05 .

\section{Results}

Fig. I shows the record obtained from Case 2 during a single Valsalva manoeuvre of 30 seconds' duration. Five individual angiograms (indicated by the upward steps in the figure) were performed during this particular Valsalva manoeuvre, in addition to the control resting angiogram. The arrows on the figure indicate which angiograms were used for volume calculation in this study. Representative angiograms from which calculations were made are shown in Fig. 2. All five patients had comparable alterations of ventricular volumes during the strain and release phases of the Valsalva manoeuvre, as shown in Table 2.

Validation of the accuracy of the present technique of angiography in calculating left ventricular volumes was accomplished by comparison of stroke volumes determined by Fick or indicator dilution methods during the control condition with those measured angiographically. Angiographic stroke volumes yielded a maximum variation of \pm 35 per cent, with mean variation of 5 per cent from those determined independently.

A summary of the means of the aortic pressure, pulse pressure, and heart rate response of all five patients during Valsalva manoeuvre is shown in Fig. 3, and the individual values are listed in Table 2. During the strain phase, the systolic blood pressure moved downwards, returning during the release phase towards normal in three of the five patients. Pulse rate rose steadily during strain and was still raised above control levels during relaxation.

During strain, mean end-diastolic volume declined nearly 50 per cent and returned by release to control levels (Fig. 4). In similar fashion, endsystolic volume declined during strain phase, but unlike end-diastolic volume did not return to pre-

TABLE 2 Measured and calculated haemodynamic and volumetric data of each of 5 patients

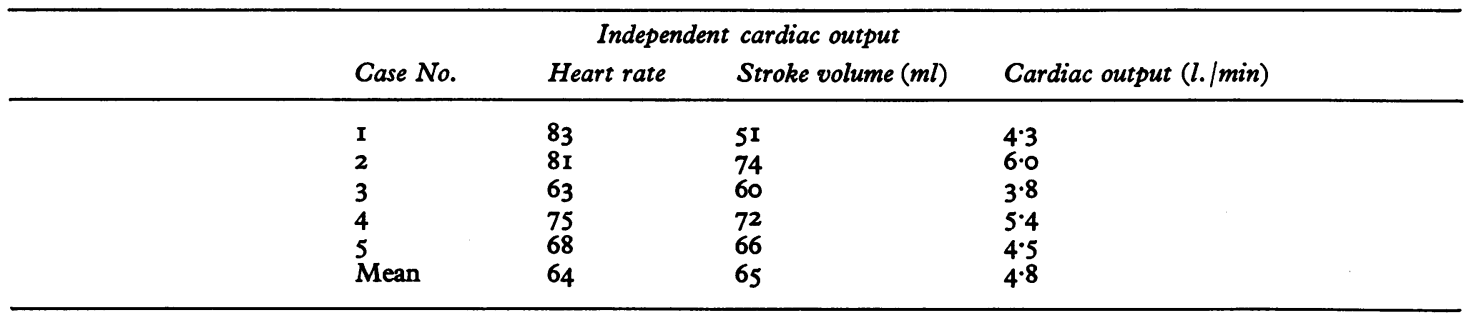

\begin{tabular}{|c|c|c|c|c|c|c|c|c|c|}
\hline \multicolumn{10}{|c|}{ Angiographic control } \\
\hline \multirow{2}{*}{$\begin{array}{l}\text { Case } \\
\text { No. }\end{array}$} & \multirow{2}{*}{$\begin{array}{l}\text { Heart } \\
\text { rate }\end{array}$} & \multicolumn{3}{|c|}{ Left ventricular volumes $(\mathrm{ml})$} & \multicolumn{3}{|c|}{ Aortic pressures $(\mathrm{mmHg})$} & \multirow{2}{*}{$\begin{array}{l}\text { Cardiac } \\
\text { output } \\
(l . / \text { min })\end{array}$} & \multirow{2}{*}{$\begin{array}{l}\text { Ejection } \\
\text { fraction }\end{array}$} \\
\hline & & $\begin{array}{l}\text { End- } \\
\text { diastolic }\end{array}$ & $\begin{array}{l}\text { End- } \\
\text { systolic }\end{array}$ & Stroke & Systolic & Diastolic & Pulse & & \\
\hline $\mathbf{I}$ & 80 & 228 & I65 & 63 & 98 & 66 & 32 & $5 \cdot 0$ & 0.28 \\
\hline 2 & 90 & 87 & 20 & 67 & 166 & 88 & 78 & $6 \cdot 0$ & 0.77 \\
\hline 3 & 70 & I 3 & 64 & 49 & 158 & 76 & 82 & $3 \cdot 4$ & 0.43 \\
\hline 4 & 75 & 160 & 86 & 74 & 106 & 68 & 38 & $5 \cdot 6$ & 0.46 \\
\hline & 77 & 83 & 34 & 49 & 122 & 66 & 56 & 3.8 & 0.59 \\
\hline Mean & 78 & I34 & 74 & 60 & 130 & 73 & 57 & $4 \cdot 8$ & 0.51 \\
\hline \multicolumn{10}{|c|}{ Strain or phase 2 of Valsalva } \\
\hline $\mathbf{I}$ & 94 & I65 & I46 & I9 & 93 & 74 & 19 & $I \cdot 8$ & 0.12 \\
\hline 2 & 105 & 45 & I3 & 32 & 124 & 87 & 37 & 3.4 & 0.71 \\
\hline 3 & 77 & 49 & 37 & 12 & 100 & 80 & 20 & 0.9 & 0.24 \\
\hline 4 & 76 & 84 & 43 & $4 I$ & II 8 & 84 & 34 & $3 \cdot I$ & 0.49 \\
\hline & 86 & 47 & I5 & 32 & 97 & 74 & 23 & $2 \cdot 8$ & 0.68 \\
\hline Mean & 88 & 78 & 51 & 27 & 106 & 80 & 27 & $2 \cdot 4$ & 0.45 \\
\hline \multicolumn{10}{|c|}{ Release or phase 4 of Valsalva } \\
\hline $\mathbf{I}$ & IO2 & 233 & I39 & 94 & 70 & 48 & 22 & $9 \cdot 6$ & 0.40 \\
\hline 2 & 128 & 82 & 13 & 69 & I74 & 96 & 78 & $8 \cdot 8$ & 0.84 \\
\hline 3 & 72 & 100 & 48 & 52 & 124 & 84 & 40 & $3 \cdot 7$ & 0.52 \\
\hline 4 & 82 & I64 & 77 & 87 & IIO & 70 & 40 & $7 \cdot I$ & 0.53 \\
\hline 5 & IO3 & 75 & 26 & 49 & 106 & 60 & 46 & $5 \cdot 0$ & 0.65 \\
\hline Mean & 97 & I3I & $6 I$ & 70 & 117 & 72 & 45 & $6 \cdot 8$ & 0.59 \\
\hline
\end{tabular}


Valsalva levels during release (Fig. 5). As can be anticipated, then, from these measured volumes, stroke volume during squeeze decreased 50 per cent, but rebounded to just above control levels upon release of straining (Fig. 6, Table 2).

Ejection fraction showed no consistent change during strain. In relaxation, however, there was a

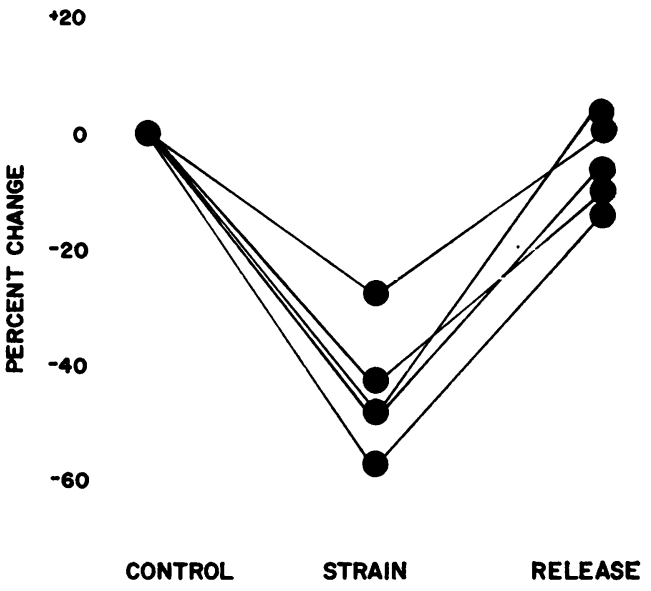

FI G. 4 This figure shows the striking reduction in left ventricular end-diastolic volume during strain phase $(P<0 \cdot 0 I)$ with rebound to control levels upon release $(P>0.05)$.

uniform and statistically significant rise over control measurements (Fig. 7).

\section{Discussion}

These angiographic studies permitted a quantitative assessment of volumetric alterations occurring during the Valsalva manoeuvre in man and confirm conclusions that were heretofore based on animal studies or extrapolations of pressure data for volume 


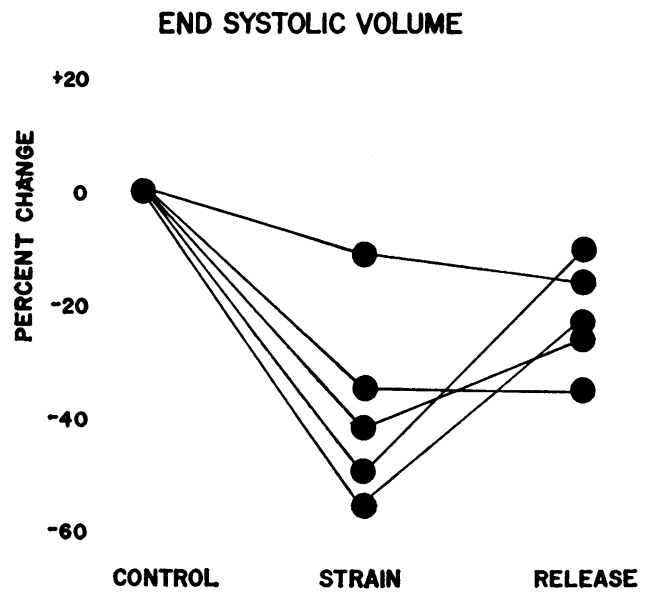

FI G.5 Left ventricular end-systolic volume diminishes during phase $2(P<0.05)$. In phase 4 , however, control volumes are not reached $(P<0.05)$.

estimation. Previous indirect data in humans are consistent with the conclusion that the fall in systemic arterial blood pressure during phase 2 results from diminished venous return, and thus, diminished cardiac output. The results of this study

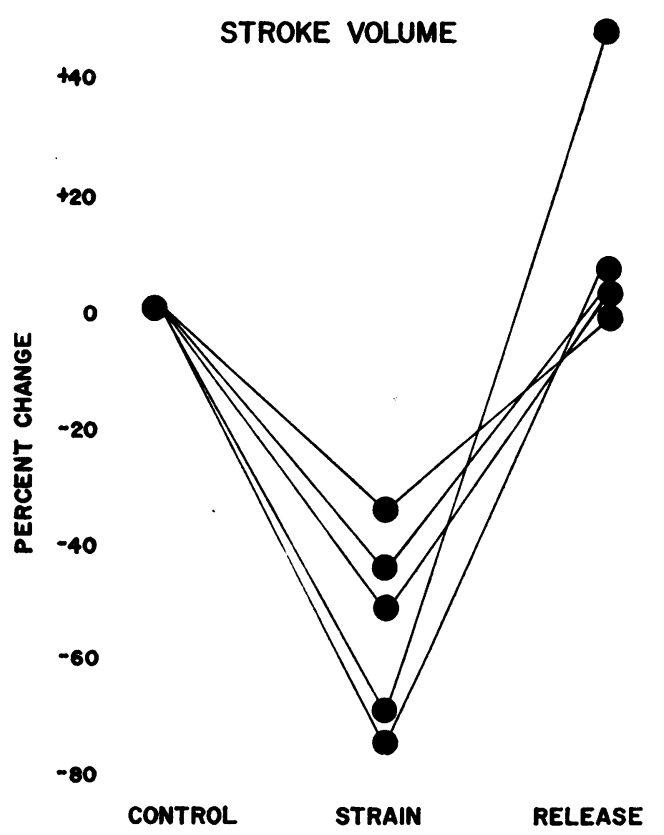

FIG. 6 Stroke volume changes in the five patients reflect the changes seen in the left ventricular enddiastolic volume and left ventricular end-systolic volume.

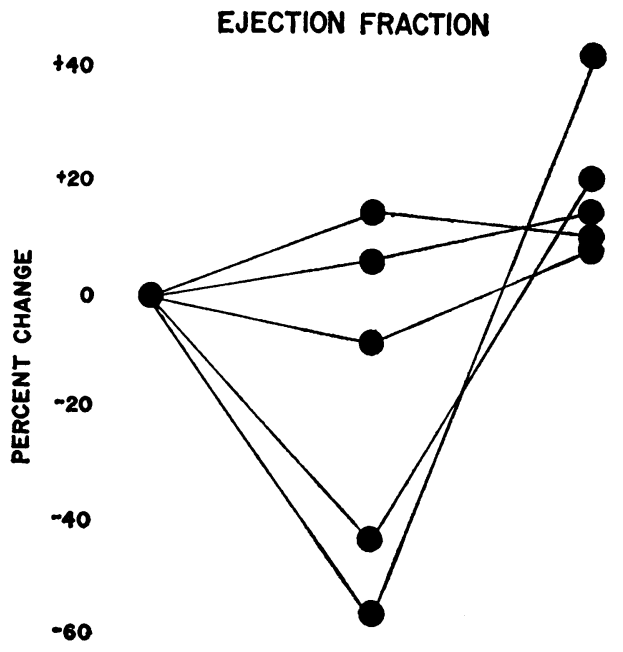

CONTROL STRAIN

RELEASE

FIG. 7 Ejection fraction changes variably during the strain phase of Valsalva, but is uniformly augmented in phase 4 after release of straining $(P<0.01)$.

support this concept by demonstrating a pronounced reduction in left ventricular end-diastolic volume and end-systolic volume which is greater than could be accounted for by heart rate change alone. Thus, the cardiac output falls, and despite the 'support' (Hamilton et al., 1936) from the raised intrathoracic pressure and raised calculated peripheral vascular resistance, arterial blood pressure falls. The fact that the systolic pressure falls more than the diastolic pressure can be explained in two ways. First, a smaller stroke volume for a given aortic pressure-volume relation would produce a smaller systolic peak pressure. Second, as the cardiac output decreases, the aorta 'empties', and thus, less pressure change is required to accommodate a given stroke volume.

In the present study, a definite overshoot in the systolic pressure was not observed after release of straining, though the pressure did tend to return towards normal. This lack of overshoot of blood pressure may be explained in two ways. The first is that since four of the patients studied did have coronary artery disease proven by previous infarction in some and significantly abnormal coronary arteriograms in all, the abnormal response was an indicator of their heart disease. The other factor which probably contributed is the cumulative effect of contrast material per se. The changes in systolic and diastolic blood pressure and cardiac output are consistent with the added effect of phase 4 Valsalva, plus the previously described effects of angiographic contrast medium on circulatory dynamics (Kloster 
et al., 1966). By the time angiograms were performed during phase 4 , a cumulative mean dose of $0.7 \mathrm{mg} / \mathrm{kg}$ of contrast medium had been administered over the preceding 7 to $22 \mathrm{sec}$.

In all the patients, despite the increased heart rate during phase 4, the left ventricular enddiastolic volume returned to control values, stroke volume exceeded control slightly, and thus, cardiac output was significantly, though transiently, increased $\mathbf{4 2}$ per cent above resting levels. The return to control left ventricular end-diastolic volume, despite the increased heart rate, is consistent with increased return of blood from the venous reservoir which had accumulated during the strain phase.

The increases in heart rate and ejection fraction during phase 4 produced a significantly increased cardiac output and are consistent with a continuing adrenergic influence on the heart. As has been noted previously, though the peripheral vascular resistance may seem to fall acutely when calculated from data measured in this changing haemodynamic situation, this classic quotient may not reflect the actual state of constriction of the resistance vessels at which adrenergic influence would be mediated. It may only represent the fact that the aorta, having previously emptied, is now lower on its pressure-volume curve (Greenfield et al., 1967). Therefore, this fall of calculated peripheral vascular resistance should not necessarily be construed as evidence countering a continuous adrenergic influence.

The use of contrast material for direct beat-bybeat determination of cardiac output and ejection fraction has in the past been limited to the resting steady-state condition. Use of this method to evaluate transient haemodynamic changes has been limited previously by the large volumes of contrast usually employed ( $\mathrm{I}-2 \mathrm{ml} / \mathrm{kg}$ ) which produce significant haemodynamic changes (Kloster et al., 1966). In the present study, by choosing patients with relatively normal-sized hearts and critically timing the injection from the patient's electrocardiogram, a much smaller dose of contrast could be employed (IO $\mathrm{ml}$ per angiogram) for adequate left ventricular visualization. Viamonte (197I) has indicated that for adequate visualization during angiography a ratio of $\mathrm{I} \mathrm{ml}$ contrast of 76 per cent concentration to 8 to Io $\mathrm{ml}$ blood is required. This ratio was attained in the four patients with normal-sized hearts. In the one exception (Case $\mathrm{r}$ ), the dose of contrast was similar to that used in the other patients, and adequate visualization was achieved.

\section{Conclusion}

In conclusion, the above study showed a significant reduction in left ventricular volume during the strain phase of the Valsalva manoeuvre (phase 2). The data are consistent with the abrupt blockade of venous return from the periphery as the basis for this fall of left ventricular volume. By similar logic, the demonstration of the return of left ventricular volume to control values, despite the increase of heart rate above control levels in that period just after release of straining (phase 4 ), seems best explained by transiently augmented venous return.

\section{References}

Alderman, E. L., Sandler, H., Brooker, J. Z., Sanders, W. J., Simpson, C., and Harrison, D. C. (1973). Light-pen computer processing of video image for the determination of left ventricular volume. Circulation, 47, 309.

Goldblatt, A., Harrison, D. C., Glick, G., and Braunwald, E. (1963). Studies on cardiac dimensions in intact, unanesthetized man. II. Effects of respiration. Circulation Research, 13, 455 .

Greenfield, J. C., Jr., Cox, R. L., Hernandez, R. R., Thomas, C., and Schoonmaker, F. W. (1967). Pressure-flow studies in man during the Valsalva maneuver with observations on the mechanical properties of the ascending aorta. Circulation, 35, 653

Hamilton, W. F., Woodbury, R. A., and Harper, H. T. (1936). Physiologic relationships between intrathoracic, intraspinal and arterial pressures. Fournal of the American Medical Association, 107, 853.

Kloster, F. E., Bristow, J. D., Jacobs, W. R., Porter, G. A., and Griswold, H. E. (1966). Hemodynamic effects of angiocardiography. 'Investigative Radiology, 1, 398.

Stone, D. J., Lyon, A. F., and Teirstein, A. S. (I965). A reappraisal of the circulatory effects of the Valsalva maneuver. American fournal of Medicine, 39, 923.

Viamonte, M., Jr. (197I). Innovations in angiography. Radiologic Clinics of North America, 9, 36r.

Requests for reprints to Dr. Donald C. Harrison, Cardiology Division, Stanford University School of Medicine, Stanford, California 94305, U.S.A. 